\title{
Estado nutricional y rendimiento académico en escolares de 7 a 14 años de la Institución Educativa Mi Jesús, Lurigancho, Lima
}

\author{
Nutritional status and academic achievement in school 7 to 14 years of "my Jesus college" La Era- \\ Lima, 2016
}

Jacksaint Saintila1*, Mery Rodríguez Vásquez²

\begin{abstract}
RESUMEN
Objetivo: Determinar la relación entre el estado nutricional y rendimiento académico en escolares de 7 a 14 años de la "Institución Educativa Mi Jesús”, La Era-Lima, 2016. Materiales y métodos: De enfoque cuantitativo, de corte transversal, de diseño no experimental y de tipo descriptiva-correlacional. La población estuvo conformada por 80 escolares de $1^{\circ}$ a $6^{\circ}$ grado de primaria y de $1^{\circ}$ a $2^{\circ}$ grado de secundaria. Las mediciones antropométricas que se realizaron para determinar el estado nutricional fueron: el peso $(\mathrm{kg})$, la talla $(\mathrm{cm})$, edad (años), Índice de Masa Corporal (IMC) y talla para la edad (T/E). Para evaluar la ingesta proteica y de minerales se aplicó un cuestionario de recordatorio de 24 horas. Para evaluar el rendimiento académico se utilizó el boletín académico de todo el año escolar. Resultados: Se evidenció que el 53\% de los estudiantes presentaron un Índice de Masa Corporal dentro de lo normal, el 61.3\% presentó una talla adecuada para la edad. También, el 70\% ha alcanzado un rendimiento adecuado (entre logro destacado y logro previsto). Finalmente, se encontró que el 56.3\% tiene una ingesta normal de proteína; referente a la ingesta de hierro, del sexo femenino, 18 presentan una ingesta normal en un 58.1\% y solamente 8 presentan una ingesta alta en un $25.5 \%$; asimismo, del sexo masculino, se observó que 25 presentan ingesta normal en un 51\% y 12 tienen una ingesta alta en un 24.5\% y el 53.8\% presenta una ingesta normal de zinc. Conclusión: Existe relación estadísticamente significativa entre el estado nutricional y rendimiento académico $(\mathrm{p}<.05)$.
\end{abstract}

Palabras clave: Estado nutricional, rendimiento académico. IMC. T/E. niños

\begin{abstract}
Objective: This research seeks to determine the relationship between nutritional status and academic achievement in 7 to 14-year-old students of Mi Jesus School, La Era, Lima, 2016. Materials and methods: A quantitative approach was used, cross-sectional design non-experimental and descriptive-correlational. Participants were 80 elementary students from 1st through 6th grade and 1st through 2nd grade secondary level. The instruments used to assess the nutritional status were an anthropometric record form (Body Mass Index and height for the age), and the assessment of protein and minerals intake was through the 24-hour recall. In addition, school records were used to assess academic performance. Results: The results showed that $53.8 \%$ of 7 to 14 -year-old students from My Jesus School had a BMI within the standard ranges. We also found that more than half (61.3\%) of students presented an adequate height for their age. $70 \%$ have achieved an adequate performance (between outstanding achievement and expected achievement). It was also demonstrated that from the entire sample, more than half (56.3\%) of students have a normal intake of protein and 53.8\% have a normal intake of iron and zinc. Conclusions: it was found that there is a statistically significant relationship between BMI, the H/A, protein intake and minerals with academic performance $(\mathrm{p}<.05)$.
\end{abstract}

Keywords: Nutritional status, academic performance. BMI. H/A. children.

${ }^{1}$ Licenciada en Nutrición Humana, Facultad de Ciencias de la Salud, Universidad Peruana Unión, Lima, Perú.
${ }^{2}$ Docente de EP Nutrición Humana, Facultad de Ciencias de la Salud, Universidad Peruana Unión, Lima, Perú. 


\section{INTRODUCCIÓN}

El estado nutricional se define como la condición en la que se encuentra el organismo, de acuerdo a los nutrientes que se consume y medido a través de los indicadores nutricionales. Cuando estos indicadores se encuentran dentro de los rangos normales, esto indica un estado nutricional adecuado, en el caso contrario, indica un estado nutricional inadecuado. En los escolares, el estado nutricional se manifiesta de manera diferente en todo el mundo, lo cual se debe a varios factores, tales como pobreza, desigualdad, deficiente educación de las madres etc., originándose así un alto índice de retardo en el crecimiento o desnutrición crónica, sobrepeso y obesidad (Marcos, 2008).

De hecho, en el año 2014 el retardo en el crecimiento afectó a 159 millones de niños y la prevalencia fue $23.8 \%$. Se ha evidenciado que 1 de cada 4 niños sufría de retraso en el crecimiento debido a la desnutrición crónica en los períodos cruciales de crecimiento y desarrollo intelectual. Por otro lado, según la Organización Mundial de la Salud (OMS), el sobrepeso y la obesidad afectaron a 41 millones de niños en el año 2014, la prevalencia fue 6.1\% (United Nations Children's Fund, 2015).

En el Perú, se ha observado un incremento (de 8,3 a 9,3\%) del retardo de crecimiento que se ha registrado a nivel urbano en la primera mitad del año 2015. También, los departamentos con más altos niveles de retardo de crecimiento como Huancavelica (44.7\%), Cajamarca (32.0\%), Apurímac (30.9), Ayacucho (30.3\%) y Cusco (29.1\%), son aquellos que presentan los más altos índices de analfabetismo. Además, el sobrepeso y la obesidad alcanzaron, en el 2014, al 9.2\% de los niños a nivel nacional según el patrón de la Organización Mundial de Salud (OMS), es decir, 1 de cada 11 niños; siendo que las regiones más afectadas por este problema son Tacna 19.2\%, Moquegua 7.1\% mientras que Lima Sur y Lima Este alcanzaron un 14.6\% (INEI, 2015; MINSA, 2012).

Por otro lado, el rendimiento académico se define como el resultado del trabajo escolar realizado por el estudiante, es decir, la cantidad de conocimientos y habilidades adquiridas por el alumno y será expresado en el promedio ponderado de las notas logradas en las asignaturas al finalizar el año escolar (5). Según la Evaluación Censal de Estudiantes (ECE-2015), en el Perú, para el año 2014, se ha observado una mejora de $20.1 \%$ en el nivel Satisfactorio y una disminución de $22.6 \%$ a nivel de Inicio en Comprensión Lectora. En cuanto a los estudiantes de las escuelas públicas, el aumento en el nivel satisfactorio se dio no solamente en comprensión lectora, sino también en
Matemática (7\% y 2\%, respectivamente). A nivel de las escuelas privadas, se registró una mejora en $4 \%$ en Comprensión Lectora. No obstante, en Matemática se observó una disminución de 1 en comparación al año 2014. Por tanto, en la ECE 2015, el porcentaje de estudiantes de nivel satisfactorio en Matemática de las escuelas públicas es más alto que el de las escuelas privadas (MINEDU, 2015).

El rendimiento académico es un proceso multifactorial que está condicionado por múltiples factores, en el niño de edad escolar, tales como estado nutricional, estado de salud, el nivel de desarrollo neuro-cognitivo y factores socioeconómicos. En muchos países en desarrollo, donde la malnutrición es generalizada, se ha visto disminuido la capacidad cognitiva de los estudiantes. De hecho, la nutrición y alimentación saludable constituyen un elemento vital e indispensable en el desarrollo del escolar, ya sea desarrollo psicológico, físico e intelectual (Haile, Nigatu, Gashaw \& Demelash, 2016; El Hioui, 2008).

Hernández (2008), en la ciudad de Lima, encontró relación estadísticamente significativa entre estado nutricional y rendimiento académico $(\mathrm{p}<.05)$. Asimismo, Ogunsile (2012), en la India, demostró que el estado nutricional está asociado con el rendimiento escolar. También, Rashmi et al. (2015), en la India encontraron que el estado nutricional está significativamente relacionado con el rendimiento escolar. Por otro lado, Izidoro et al, (2014) en Brasil, demostraron que una correlación estadísticamente significativa entre el estado nutricional y el rendimiento académico $(\mathrm{p}<0.05)$. Más aun, Sarma et al, (2013) realizaron un estudio en Sri Lanka, concluyendo que hubo una correlación significativa entre estado nutricional y rendimiento académico. Igualmente, Murillo et al. (2012) elaboraron una investigación en Ecuador, observando que la nutrición influye en el rendimiento escolar. Por otra parte, Ejekwu et al. (2012) efectuaron un estudio en Nigeria, observando a los niños que presentaron desnutrición obtuvieron un bajo rendimiento académico. Además, Márquez et al, (2009) llevaron a cabo un estudio en México, concluyendo que los estudiantes que no tuvieron un estado nutricional, dentro de los rangos, presentaron disminución en su rendimiento académico. Asimismo, Arzapalo et al, (2011) realizaron un estudio en Lima, evidenciando que los escolares que tuvieron un estado nutricional en delgadez presentaron un rendimiento académico en proceso. De igual manera, Ruíz (2006) realizó un estudio en Venezuela, encontrando que la suplementación de hierro en escolares podría ser útil para incrementar el rendimiento académico. Finalmente, Haile et al (2016), en un estudio desarrollado en Etiopia, mostraron que existe 
correlación estadísticamente significativa entre el estado de nutricional y rendimiento académico.

Naik et al. (2015) desarrollaron un estudio en la India, no encontraron correlación entre el estado nutricional y el rendimiento académico. Figuerola, (2015) realizó un estudio en España, evidenciando que no hubo correlación estadísticamente significativa entre el estado de nutricional y el rendimiento académico. Finalmente, González et al (2013) realizaron un estudio en Colombia, no encontrando correlaciones significativas entre el IMC y el rendimiento académico.

La evaluación nutricional y la vigilancia del crecimiento físico e intelectual, de los niños, constituyen una de las acciones más relevantes a cumplir en atención primaria. La evaluación del estado nutricional es esencial para determinar los requerimientos nutricionales y prevenir posibles patologías asociadas a deficiencias de nutrientes y así favorecer el crecimiento y el desarrollo adecuado de las funciones cognoscitivas del escolar Ejekwu et al. (2012). Los indicadores que son de mayor utilidad, para valorar el estado de nutrición, son: peso, talla, perímetro craneal, perímetro del brazo y el grosor del pliegue cutáneo, entre otros. Asimismo, los índices de relación que han demostrado ser de mayor utilidad, para valorar el estado de nutrición, son: peso para talla $(\mathrm{P} / \mathrm{T})$, talla para la edad (T/E), peso para la edad (P/E) y el Î́ndice de Masa Corporal (IMC) (El Hioui, 2008).

El Ministerio de Educación (MINEDU) ha elaborado un Diseño Curricular Nacional de la Educación Básica Regular, con la finalidad de mejorar la calidad educativa, formar estudiantes críticos, creativos, responsables y solidarios, asimismo, acompañar a los docentes en sus procesos pedagógicos. Este documento contiene los aprendizajes que deben alcanzar cada estudiante, según su nivel educativo en cualquier lugar del país (MINEDU, 2009).

El rendimiento académico se mide en escala ordinal, según a las siguientes categorías (MINEDU, 2012):

Logro destacado: cuando el estudiante evidencia el logro de los aprendizajes previstos, demostrando, incluso, un manejo adecuado y satisfactorio en todas las tareas propuestas en un tiempo programado.

Logro previsto: cuando el estudiante evidencia el logro de los aprendizajes previstos en el tiempo.

En proceso: cuando el estudiante está en camino de lograr los aprendizajes previstos, para lo cual requiere acompañamiento durante un tiempo razonable para lograrlo.
En inicio: cuando el estudiante está en camino de lograr los aprendizajes previstos o evidencia dificultad para el desarrollo de estos, necesitando mayor tiempo de acompañamiento e intervención del docente.

Diversas investigaciones han demostrado que el estado nutricional está estrechamente relacionado con el desarrollo físico y de las capacidades intelectuales, las cuales influyen en el rendimiento académico del niño de edad escolar. De hecho, la relación entre el estado nutricional y el rendimiento académico ha sido de interés para muchos investigadores en el mundo científico desde hace varias décadas en los países en desarrollo, los resultados han evidenciado que la talla para la edad, lo cual es un indicador del retardo de crecimiento o desnutrición crónica afecta el crecimiento físico, la morbimortalidad, el desarrollo de las capacidades cognoscitivas, la reproducción y la fuerza productiva (Ateillah, Aboussaleh, Rachid \& Ahami, 2012; Sarma et al. 2013).

Razón por la cual la nutrición y la educación de calidad figuran en un lugar preponderante entre los 17 objetivos de desarrollo sostenible (ODS) que se aprobaron en la Agenda hasta el 2030 para el desarrollo sostenible por los estados miembros de la Organización de las Naciones Unidas (ONU) (UN, 2015).

Ante esta preocupación, decidimos realizar este estudio, con el propósito de determinar la relación entre el estado nutricional y el rendimiento académico, para conocer y evitar la consecuencias que tiene una nutrición inadecuada en el desarrollo de las funciones cognitivas del estudiante, enriquecer el conocimiento local y fortalecer la importancia del trabajo del profesional de nutrición como parte del quehacer en el control de desarrollo y crecimiento a través de la fomentación de hábitos basados en el eje temático de alimentación y nutrición saludable y promoción de estilos de vida saludables, a fin de mejorar el estado nutricional de la población infantil.

\section{MATERIAL Y MÉTODOS}

Esta investigación es de enfoque cuantitativo, de corte transversal, de diseño no experimental y de tipo descriptivo correlacional. Se trabajó con una muestra conformada por 80 niños entre 7 y 14 años de la Institución Educativa Mi Jesús, La Era -Lima. Además, se seleccionó a los participantes mediante el muestreo no probalístico por conveniencia.

La distribución sociodemográfica, de los estudiantes, muestra que el $61.3 \%$ es de género masculino y el 
$38.8 \%$ de género femenino. Asimismo, respecto a la procedencia, el $60 \%$ es de la costa y el $40.1 \%$ procede de la selva, sierra y del extranjero. Del mismo modo, en cuanto a la filiación religiosa, el $75 \%$ es adventista y el $25.1 \%$ está entre católico, evangélico y otros. Por otro lado, el 78.7\% tiene un nivel primario y el 21.3\% está en un nivel segundario. Finalmente, de acuerdo a la edad, el 57.5\% de los participantes comprende entre la edad de 7 y 10 años, el $42.6 \%$ entre las edades de 10 y 14 años.

Los instrumentos que se utilizaron, para evaluar el estado nutricional, fue la ficha de registro antropométrico (Índice de Masa Corporal y Talla para la edad), y la valoración de la ingesta dietética fue mediante el recordatorio de 24 horas. Además, se utilizó el boletín académico de todo el año escolar para evaluar el rendimiento académico. Asimismo, los datos obtenidos de la evaluación antropométrica fueron comparados con la Valoración Nutricional Antropométrica del Centro Nacional de Alimentación y Nutrición (CENAN) adaptado de la OMS, 2006 y Tabla de IMC Para la edad, de niños de 5 a 18 años adaptado de la OMS, 2007. Además, se clasificó el rendimiento académico de cada escolar, según la escala de clasificación establecida por el Ministerio de Educación (MINEDU). Los datos obtenidos fueron ingresados en una hoja de cálculo de Excel en su versión 2013; además, se utilizó el programa SPSS en su versión 22 para analizar los datos del estudio, el mismo que facilitó el análisis de las variables de estudio. Para realizar el análisis descriptivo, se utilizó tablas de frecuencia y porcentaje y para realizar el análisis de correlacional, se utilizó el estadístico del Chi Cuadrado.de correlación Tau-b de Kendall.

\section{RESULTADOS}

Tabla 1

Distribución de la muestra según la ingesta proteica de los escolares

\begin{tabular}{lll}
\hline & $\mathrm{N}$ & $\%$ \\
\hline Ingesta baja & 16 & 20.0 \\
Ingesta normal & 45 & 56.3 \\
Ingesta alta & 19 & 23.8 \\
Total & 80 & 100.0 \\
\hline
\end{tabular}

Según se observa la distribución de la ingesta proteica, en la Tabla 1, del 100\% de los evaluados el 56.3\% de los participantes presenta ingesta proteica normal, el 23.8\% una ingesta de proteína alta y el $20.0 \%$ tiene ingesta proteica baja, basado en las recomendaciones nutricionales establecidas.
Tabla 2

Distribución de la muestra según la ingesta de hierro por género

\begin{tabular}{clll}
\hline Sexo & & $\mathrm{N}$ & $\%$ \\
\hline Masculino & Ingesta baja & 12 & 24.5 \\
& Ingesta normal & 25 & 51.0 \\
& Ingesta alta & 12 & 24.5 \\
& Total & 49 & 100.0 \\
Femenino & Ingesta baja & 5 & 16.1 \\
& Ingesta normal & 18 & 58.1 \\
& Ingesta alta & 8 & 25.8 \\
& Total & 31 & 100.0 \\
\hline
\end{tabular}

Al observar la Tabla 2, se evidencia que el número de los estudiantes de sexo femenino que presentan una ingesta normal de hierro es 18, en una proporción de $58.1 \%$, y la cantidad que tiene una ingesta alta es solamente 8, en una proporción de $25.5 \%$; asimismo, referente al sexo masculino, se observa que 25 de los estudiantes presentan ingesta normal de hierro, en un 51\%, y 12 tienen una ingesta alta, en una proporción de $24.5 \%$.

Tabla 3

Distribución de la muestra según la ingesta de zinc de los escolares

\begin{tabular}{lll}
\hline & $\mathrm{N}$ & $\%$ \\
\hline Ingesta baja & 17 & 21.3 \\
Ingesta normal & 43 & 53.8 \\
Ingesta alta & 20 & 25.0 \\
Total & 80 & 100.0 \\
\hline
\end{tabular}

Según se ha observado en la Tabla 3, el 53.8\% de los participantes presentan una ingesta normal de Zinc, el 25.0\% tiene una ingesta alta y el 21.3\% una ingesta baja, según las recomendaciones establecidas por los expertos.

Tabla 4

Distribución de la muestra según el Índice de masa corporal de los estudiantes

\begin{tabular}{lll}
\hline Diagnóstico de IMC & $\mathrm{N}$ & $\%$ \\
\hline Delgadez & 9 & 11.3 \\
Normal & 43 & 53.8 \\
Sobrepeso & 8 & 10.0 \\
Obesidad & 20 & 25.0 \\
Total & 80 & 100.0 \\
\hline
\end{tabular}


Al analizar el estado nutricional, según el IMC de los escolares en la Tabla 4, se observó que el mayor porcentaje de los niños presentan un Índice de Masa Corporal dentro de los parámetros normales con un $53.8 \%$, mientras que el $46.3 \%$ presenta un estado nutricional fuera de los rangos normales.

\section{Tabla 5}

Distribución de la muestra según la talla para la edad de los estudiantes de 7 a 14 años de la I.E. Mi Jesús

\begin{tabular}{lll}
\hline Evaluación de Talla & $\mathrm{N}$ & $\%$ \\
\hline Talla normal & 49 & 61.3 \\
Retardo de crecimiento & 31 & 38.8 \\
Total & 80 & 100.0 \\
\hline
\end{tabular}

En la Tabla 5 se muestra la distribución de la talla para la edad de los estudiantes, la cual refiere que el $61.3 \%$ presenta una talla normal para la edad y solo el $38.8 \%$ presenta retardo en el crecimiento.

\section{Tabla 6}

Distribución de la muestra según la ingesta dietética de los escolares de 7 a 14 años de la I.E. Mi Jesús

\begin{tabular}{lll}
\hline Ingesta dietética & $\mathrm{N}$ & $\%$ \\
\hline Adecuada & 44 & 55.0 \\
Inadecuada & 36 & 45.0 \\
Total & 80 & 100.0 \\
\hline
\end{tabular}

Según se observa la distribución de la ingesta dietética en la Tabla 6, de un 100\%, el 55\% de los participantes presenta buena ingesta dietética y el $45 \%$ tiene una ingesta inadecuada.

Tabla 7

Distribución de la muestra según la escala de la clasificación del rendimiento académico de los estudiantes de 7 a 14 años de la I.E. Mi Jesús.

\begin{tabular}{lll}
\hline Rendimiento escolar & $\mathrm{N}$ & $\%$ \\
\hline Inicio & 1 & 1.3 \\
Proceso & 23 & 28.7 \\
Logro previsto & 26 & 32.5 \\
Logro destacado & 30 & 37.5 \\
Total & 80 & 100.0 \\
\hline
\end{tabular}

En la Tabla 7 se muestra la distribución del rendimiento escolar de los estudiantes de la I.E Mí Jesús, la cual refiere que el $37.5 \%$ está en la categoría de logro destacado, el 32.5\% en la categoría de logro previsto y el $28.7 \%$ está en la categoría de proceso. Solo se ha encontrado un $1.3 \%$ en inicio o en dificultad para de lograr los aprendizajes previstos.

Tabla 8

Análisis de relación entre el Índice de Masa Corporal y el rendimiento académico

\begin{tabular}{llll}
\hline & Valor & $\mathrm{gl}$ & $\mathrm{p}$ \\
\hline Chi-cuadrado de Pearson & 28,862 & 9 &, 001 \\
$\mathrm{~N}$ & 80 & & \\
\hline
\end{tabular}

La Tabla 8 describe la relación entre el estado nutricional y el rendimiento académico de los escolares de 7 a 14 años de la I.E. Mi Jesús según el IMC; se observó que existe relación estadísticamente significativa entre los niveles de IMC y rendimiento académico en los estudiantes del colegio Mí Jesús $(\mathrm{p}<.05)$.

Tabla 9

Distribución de relación entre los valores de la talla para la edad y el rendimiento escolar

\begin{tabular}{llll}
\hline & Valor & $\mathrm{gl}$ & $\mathrm{p}$ \\
\hline Chi-cuadrado de Pearson & 24,011 & 3 &, 000 \\
$\mathrm{~N}$ & 80 & & \\
\hline
\end{tabular}

En la Tabla 9 se observa que existe relación estadísticamente significativa entre los valores de la talla para edad y el rendimiento escolar en los estudiantes del Colegio Mi Jesús ( $\mathrm{p}<.05)$.

Tabla 10

Distribución de relación entre la ingesta dietética y el rendimiento académico

\begin{tabular}{llll}
\hline & Valor & $\mathrm{gl}$ & $\mathrm{p}$ \\
\hline Chi-cuadrado de Pearson & 15,040 & 3 &, 002 \\
$\mathrm{~N}$ & 80 & & \\
\hline
\end{tabular}

En la Tabla 10 se muestra que existe relación estadísticamente significativa entre la ingesta dietética y el rendimiento escolar del Colegio Mi Jesús $(\mathrm{p}<.05)$.

\section{DISCUSIÓN}

El estado nutricional adecuado está estrechamente relacionado con el crecimiento corporal y el desarrollo 
de las capacidades cognoscitivas y, por consiguiente, con el rendimiento académico, especialmente en la edad escolar. También, las investigaciones hacen referencia al impacto del estado nutricional en el sistema inmune o resistencia a las enfermedades y en las capacidades de aprendizajes (Ateillah, Aboussaleh, Rachid, \& Ahami, 2012).

Según la escala de clasificación del rendimiento académico, se evidenció que el $70 \%$ de los participantes se encuentran en la categoría de un rendimiento normal (logro destacado y previsto). Muy cercano a nuestros resultados, Ogunsile (2012) señaló que el 50\%, de su población de estudio, tuvo un buen rendimiento académico. No obstante, Arzapalo et al. (2011), en su estudio sobre relación entre el estado nutricional y el rendimiento académico, se comprobó que el 57.46\% de los escolares mostraron un logro destacado. De la misma forma, estos resultados coinciden con el mencionado por Izidoro et al. (2014) quienes encontraron que el 74.6\%, de los niños prescolares, fue calificada en la categoría baja. Ambos estudios ponen énfasis en otros factores que, además de los indicadores antropométricos, podrían afectar el rendimiento académico tales como: desintegración familiar y un entorno socioeconómico y cultural desfavorable. Ya que hasta ahora no ha sido posible observar a los niños en su vida diaria y en su relación con sus padres en el hogar, dos aspectos importantes dentro de las causas de un bajo rendimiento académico.

Al determinar la relación entre el IMC y rendimiento académico, se observó que existe correlación estadísticamente significativa entre sí $(p<.05)$. Conviene subrayar que el $53.8 \%$ de los participantes tienen un IMC dentro de lo normal, De igual modo, Izidoro et al. (2014) encontraron que el $76.3 \%$ de los escolares que participaron en su estudio son eutróficos, el 13\% tenía sobrepeso y solo el $1.7 \%$ estaba desnutrido. Algo parecido sucede también con los hallazgos de Márquez et al. (2009) quienes encontraron que el $88 \%$, de su población de estudio, tenía un estado nutricional normal, de la misma forma estos resultados coinciden con Murillo et al. (2012) donde encontraron que el 58\% presentó un IMC dentro de lo normal; estas tres investigaciones mostraron correlación significativamente entre el IMC y el rendimiento académico $(\mathrm{p}<.05)$. Al contrario, Figuerola (2015) encontró que el 75.3\% tenía un peso saludable. En este estudio no se encontró correlación estadísticamente significa con rendimiento académico, debido al tamaño de la muestra. Asimismo, Gonzales et al. (2013), además de evidenciar que el $40.38 \%$ de su población de estudio tenían un peso normal, encontraron que el
IMC no está significativamente correlacionado con el rendimiento escolar $(r=, 456 ; p=0,000)$.

Al observar la relación entre la T/E y el rendimiento académico, los resultados mostraron que existe correlación estadísticamente significativa entre ambos variables $(\mathrm{p}<.05)$. Tal como se observó en la tabla 13, el $61.3 \%$ presenta talla para la edad adecuada. Los resultados mencionados son semejantes a lo reportado por Sarma et al. (2013), donde el 68\% presentó una T/E adecuada. Asimismo Ejekwu et al. (2012) encontraron resultados similares, donde el $73 \%$ de los niños presentaron una talla normal para la edad. Por otro lado, Rashmi et al. (2015) encontraron que un $93 \%$ los niños presentaron una talla para la edad. En los tres estudios se ha evidenciado que hubo una correlación significativa entre la $\mathrm{T} / \mathrm{E}$ y el rendimiento académico $(\mathrm{p}<.05)$. Estos estudios difieren con Naik et al. (2015) quienes encontraron que el 51.8\% de su población de estudio presentaron retardo en el crecimiento. Por otro lado, los mismos autores, Naik et al. (2015), en otro estudio, encontraron que el $60 \%$ presentó desnutrición crónica, aguda y global; ninguno de estos resultados mostraron relación estadísticamente significativa con el rendimiento académico $(\mathrm{p}<.05)$.

Referente a la relación entre la ingesta proteica recomendada y el rendimiento académico de los estudiantes, se demostró que existe relación estadísticamente significativa entre las dos variables $(\mathrm{p}<.05)$. Sobre este punto, Perales (2016) encontró que el $91.5 \%$ de niños con rendimiento destacado y el $72.7 \%$ de niños con rendimiento en proceso consumían las cantidades de proteínas recomendadas, en este estudio se evidenció una asociación significativa entre los niños con rendimiento académico destacado y la ingesta recomendada de proteínas. La ingesta de proteínas en los estudiantes fue comparada según las recomendaciones establecidas por la OMS según la edad y el sexo de cada estudiante, clasificando de esta manera en varones: < de 27 g/día, ingesta baja; entre $27 \mathrm{~g} /$ día a $43 \mathrm{~g} /$ día, ingesta normal y > de 43 g/día, ingesta alta. En mujeres, <de 27 g/día, ingesta baja; entre 27 g/día a 44 g/día, ingesta normal y $>$ a $44 \mathrm{~g} /$ día, ingesta proteica alta. Asimismo, referente a la ingesta proteica, Ibáñez (2015) afirma que la malnutrición proteica, en la etapa escolar, afecta al crecimiento del cerebro y a su desarrollo posterior. Tiene como consecuencias alteraciones en las funciones cognitivas. Además, la calidad y la cantidad de la proteína que se ingiere influyen en el buen funcionamiento del cerebro.

Respecto a la relación a ingesta de hierro y el rendimiento académico de los estudiantes, se encontró 
que existe relación estadísticamente significativa entre la ingesta de hierro y el rendimiento académico $(\mathrm{p}<.05)$. Nuestros resultados concuerdan con Ruiz (2006) quien, en su estudio sobre la ingesta de hierro y su relación con la función cognitiva, encontró correlación estadísticamente significativa $(\mathrm{p}<.0 .5)$. Asimismo, se observó que el número de los estudiantes de sexo femenino que presentan una ingesta baja de hierro es 5 en una proporción de 16.1\% en comparación al sexo masculino, donde 12 presentan una ingesta alta de hierro en una proporción de $24.5 \%$. Cifras superiores se ha observado en un estudio realizado por Fernández et al. (2007), donde el 22.9\% de su población de estudio, de género femenino, presentó deficiencia de hierro. En un estudio realizado en la población española, por Trave et al. (2002) se ha observado que un $12.6 \%$ de las mujeres, de las adolescentes (10 a 14 años), presentaban deficiencia de hierro. De hecho, se considera que existe una serie de factores que incrementarían el riesgo y/o explicarían la mayor prevalencia de carencia de hierro, tanto en los hombres mayores de 12 años, como en las mujeres mayores de 10 años. Por un lado, se cree que la aceleración de la velocidad de crecimiento provoca una seria de cambios en la composición corporal, incluyendo un aumento de la masa magra, de la volemia y la masa eritrocitaria, además de una mayor actividad física incrementarían las pérdidas urinarias de hierro. Por otro lado, en las mujeres, las pérdidas de hierro se dan al inicio de la menarquia; de hecho, se sabe que la deficiencia de hierro estaría condicionado por las pérdidas menstruales (Sen, A., \& Kanani, 2006). Asimismo, existen factores dietéticos que podrían involucrar en esa deficiencia de hierro debido a que las preferencias alimentarias de las mujeres, en esta etapa de la menarquia, no son iguales a las de los varones; ya que la mujer se preocupa por su imagen corporal (Fernández et al. 2007), Asimismo, se comparó la ingesta de hierro de los participantes con las recomendaciones establecidas por los expertos de FAO/OMS/UNU (OMS/FAO/UNU, 1985) según la edad y el sexo. < De $10 \mathrm{mg} /$ día, ingesta baja; de $10 \mathrm{al}$ $15 \mathrm{mg} /$ día, ingesta normal y > a $15 \mathrm{mg}$ /día, ingesta alta.

En cuanto a la ingesta de zinc, según se ha observado, el 53.8\% de los participantes presentan ingesta normal. Además, se evidenció relación entre la ingesta de zinc y el rendimiento académico $(\mathrm{p}<.05)$. También el zinc es un micronutriente esencial para la neurogénesis, la migración neuronal y la sinaptogénesis. Dicho brevemente, este nutriente está relacionado con las funciones motoras, cognitivas y psicosocial en niños edad escolar. Asimismo, la deficiencia de zinc puede alterar el desarrollo cognitivo por disminución de atención y actividad motora (Karami, M, et al.
2013). La ingesta de zinc, recomendada por la OMS, se clasifica de la siguiente manera: < de $10 \mathrm{mg} /$ día, ingesta baja; de 10 al $15 \mathrm{mg} /$ día, ingesta normal y $>$ a 15 mg/día, ingesta alta (OMS/FAO/UNU, 1985).

Se concluyó que el 53.8\% de los estudiantes, de 7 a 14 años de la I.E Mi Jesús, presentaron un IMC dentro los rangos normales.

Al determinar la T/E, se encontró que más de la mitad (61.3\%) de los estudiantes presentaron una talla adecuada para su edad. Se encontró correlación significativa entre el IMC, talla para la edad y el rendimiento académico. Se observó que el 56.3\% tiene una ingesta normal de proteína.

De igual manera, se evidenció que, del sexo femenino, 18 presentan una ingesta normal de hierro en un $58.1 \%$ y solamente 8 presentan una ingesta alta en un 25.5\%; asimismo, del sexo masculino, se observó que 25 presentan ingesta normal en un 51\% y 12 tienen una ingesta alta en un $24.5 \%$; referente a la ingesta de zinc se encontró que el 53.8\% presenta una ingesta normal, alcanzando los requerimientos nutricionales establecidos por OMS/FAO/UNU (1985).

Al evaluar el rendimiento académico, según la escala de clasificación del MINEDU (2012), la mayor proporción (70\%) de los estudiantes han alcanzado un rendimiento académico adecuado (entre logro destacado y logro previsto). Se encontró relación significativa entre la ingesta proteica y de mineral con el rendimiento académico. La gran mayoría de los estudiantes alcanzaron un rendimiento académico adecuado (entre logro destacado y logro previsto). Se encontró que existe correlación estadísticamente significativa entre el estado nutricional y rendimiento académico $(\mathrm{p}<.05)$.

Se recomienda implementar un programa de promoción de la salud, con el fin de capacitar en temas relacionados a la nutrición y la alimentación saludable a los estudiantes, profesores y padres de familia de la I.E Mi Jesús para mejorar el estado nutricional de aquellos niños que presentan retardo en el crecimiento, sobrepeso y obesidad y, poniendo énfasis sobre la importancia de una alimentación balanceada y equilibrada en la función cognitiva.

\section{Declaración de financiamiento y de conflicto de intereses:}

El estudio fue financiado por los autores, quienes declaran no tener algún tipo de conflicto de interés en la investigación realizada. 


\section{Correspondencia:}

Jacksaint Saintila. Facultad Ciencias de la Salud. Universidad Peruana Unión, Carretera Central Km.
19.5 Ñaña, Lima, Perú.

e-mail: saintilajacksainti@upeu.edu.pe

\section{REFERENCIAS BIBLIOGRÁFICAS}

Arzapalo, F., Pantoja, K., Romero, J., \& Farro, G. (2011). Estado nutricional y rendimiento escolar de los niños de 6 a 9 años del Asentamiento Humano Villa RicaCarabayllo, Lima-Peru 2011. Rev. enferm. herediana, 4(1), 20-26.

Ateillah, K., Aboussaleh, Y., Rachid, S., Ahami, \& S. (2012). Evaluation nutritionnelle et son impact sur la performance scolaire des écoliers ruraux de la région de sidi Taybi dans la province de Kenitra (MAROC). Antropo, 28, 71-76.

Ejekwu AD, Ene-Obong HN, Oguizu OJ. (2012). Nutritional status and cognitive performance among children aged 5-12 from urban and rural areas of Enugu state Nigeria. Afr J Psychol Study Soc Issues. 15:481-496.

El Hioui, M., Soualem, A., Ahami, A. O. T., Aboussaleh, Y., Rusinek, S., \& Dik, K. (2008). Caractéristiques sociodémographiques et anthropométriques en relation avec la performance scolaire dans une école rurale de la ville de Kenitra (Maroc). Antropo, (17), 24-33.

Fernández, A., Troncoso, L., \& Nolberto, V. (2007). Estado de nutrición en hierro en una población de 4 a 14 años, urbano marginal, de Lima. En Anales de la Facultad de Medicina. UNMSM. Facultad de Medicina. p. 136-142.

Garcia R. (2011). Estado nutricional y rendimiento académico relacionados con el consumo de del refrigerio escolar de los niños y niñas de la escuela fiscal mixta Alberto Flores del Cantón Guaranda provincia de Bolívar. Ecuador.

Gómez, N. (2015). Relació entre els resultats acadèmics, els hàbits d'alimentació i l'estat ponderal en alumnes de primària d'escoles ubicades en municipis adherits al Programa Thao-Salut Infantil. Tesis de licenciatura. Universidad de Lérida.

González, E., \& Ortega, A. (2013). Relación de sobrepeso y obesidad con nivel de actividad física, condición física, perfil psicomotor y rendimiento escolar en población infantil (8 a 12 años) de Popayán. Revista Movimiento Cientifico, 7(1), 71-84.

Haile, D., Nigatu, D., Gashaw, K., \& Demelash, H. (2016). Height for age $\mathrm{z}$ score and cognitive function are associated with Academic performance among school children aged 8-11 years old. Archives of Public Health, 74, 17. http://doi.org/10.1186/s13690-016-0129-9

Hernández, J. (2008). Relación entre el estado nutricional y rendimiento escolar en niños de 6 a 12 años de edad de la I.E. Huáscar $N^{\circ}$ 0096. 2008. Lima: Universidad Nacional Mayor de San Marcos.

Ibáñez, E. (2009). Nutrientes y función cognitiva. NutrHosp Suplementos. 2(2):3-12.

INEI. (2015). Encuesta demógrafica y de salud familiar (ENDES). Resultados preliminares. Lima, Perú.
Izidoro, G., Santos, J. N., Oliveira, T., \& Martins-Reis, V. (2014). The influence of nutritional status on school performance. Revista CEFAC, 16(5), 1541-1547.

Karami, M, et al. (2013). The Effect of Zinc Supplementation of Lactating Rats on Short-Term and Long-Term Memory of Their Male Offspring. Health PromotPerspect, 3(2): 242-245

Marcos, C. (2008). Estado nutriconal y orientación nutricional en estudiantes de ballet de nivel elemental (parte II). Trastornos de la conducta alimentaria, 870902

Márquez, A. (2009). Estado nutricional y su rendimiento escolar en adolescentes de 12-16 años del sur de cd Obregón, son., México. Salus, 9(2).

MINEDU. Gobierno del Perú. (2009). Diseño Curricular Nacional de Educación Básica Regular. Perú.

MINEDU. Gobierno del Perú. (2012). Escala de clasificación de los aprendizajes en la Educación Básica Regular. Oficio Nº 005-2011-ME/SG-OTD-AAC. Lima, Perú.

MINEDU. Gobierno del Perú. (2015). Resultado de la Evaluación Censal de Estudiantes. Lima, Perú.

MINSA. Gobierno del Perú. (2012). Un gordo problema: sobrepeso y obesidad en el Perú. Lima, Perú.

Murillo, M., y Zambrano M. (2012). Estado nutricional con el rendimiento académico de los niños de 2do, 3ro y 4to año de básica en la escuela fiscal mixta charapoto de la parroquia charapoto, cantón sucre durante el periodo de enero-junio del 2012. Manabí - Ecuador.

Naik, S., Itagi, S., \& Patil, M. (2015). Relationship between nutritional status, intelligence and academic performance of Lambani school children of Bellary district, Karnataka. International Journal of Farm Sciences, 5(3), 259-267.

Naik, S., Itagi, S., \& Patil, M. (2015). Relationship between nutritional status and academic achievement of lambani school children. International Journal of Recent Scientific Research Vol. 6, Issue, 3, pp.3235-3238.

Nations Unis (UN). (2015). Transformer notre monde: le Programme de développement durable à l'horizon 2030 Résolution adoptée par l’Assemblée Générale. New York.

Navarro, R. (2003). El rendimiento académico: concepto, investigación y desarrollo. REICE: Revista Electrónica Iberoamericana sobre Calidad, Eficacia y Cambio en Educación.

Ogunsile, S. E. (2012). The Effect of Dietary Pattern and Body Mass Index on the Academic Performance of Inschool Adolescents. International Education Studies, 5(6),65. 
OMS/FAO/UNU. (1985). Necesidades de Energía y de Proteínas. Informes de una Sesión Consultiva Conjunta de Expertos. Series Informes Técnicos N ${ }^{\circ} 724$, Ginebra: OMS.

Perales, P. (2016). Perfil nutricional y rendimiento académico en niños preescolares que asisten a la Cuna Jardín Carmen Alto-Comas 2015. 2016.

Rashmi, M. R., Shweta, B. M., Fathima, F. N., Agrawal, T., Shah, M., \& Sequeira, R. (2015). Prevalence of Malnutrition and Relationship with Scholastic Performance among Primary and Secondary School Children in Two Select Private Schools in Bangalore Rural District (India). Indian journal of community medicine: official publication of Indian Association of Preventive \& Social Medicine, 40(2), 97.

Ruíz, A. (2006). Deficiencia de hierro en niños escolares y su relación con la función cognitiva. 10(2):10-16.
Sarma, M., Wijesinghe, D. G. N. G., \& Sivananthawerl, T. (2013). The nal Performance of Primary School Children in the Plantation Sector in Nuwara Eliya Educational Zone. Tropical Agricultural Research, 24(3).

Sen, A., \& Kanani, S. J. (2006). Deleterious functional impact of anemia on young adolescent school girls. Indian pediatrics, 43(3), 219-26.

Trave, T. D, et al. (2002). Carencia de hierro en adolescentes (10-14 años). Atención primaria. 29, 72-78.

United Nations Children's Fund (UNICEF)/World Health Organization (WHO)/World Bank Group.(2015). Levels and trends in child malnutrition. Keyfidings of the 2015 edition.

Recibido: 13/10/2016 Aceptado: 29/11/2016 